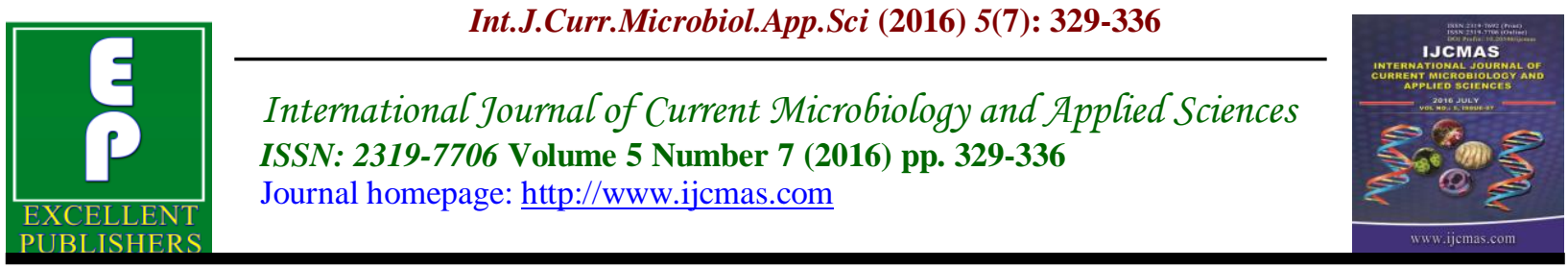

Original Research Article

http://dx.doi.org/10.20546/ijcmas.2016.507.035

\title{
Influence of Micronutrients and Growth Regulators on Shelf-life of Cabbage
}

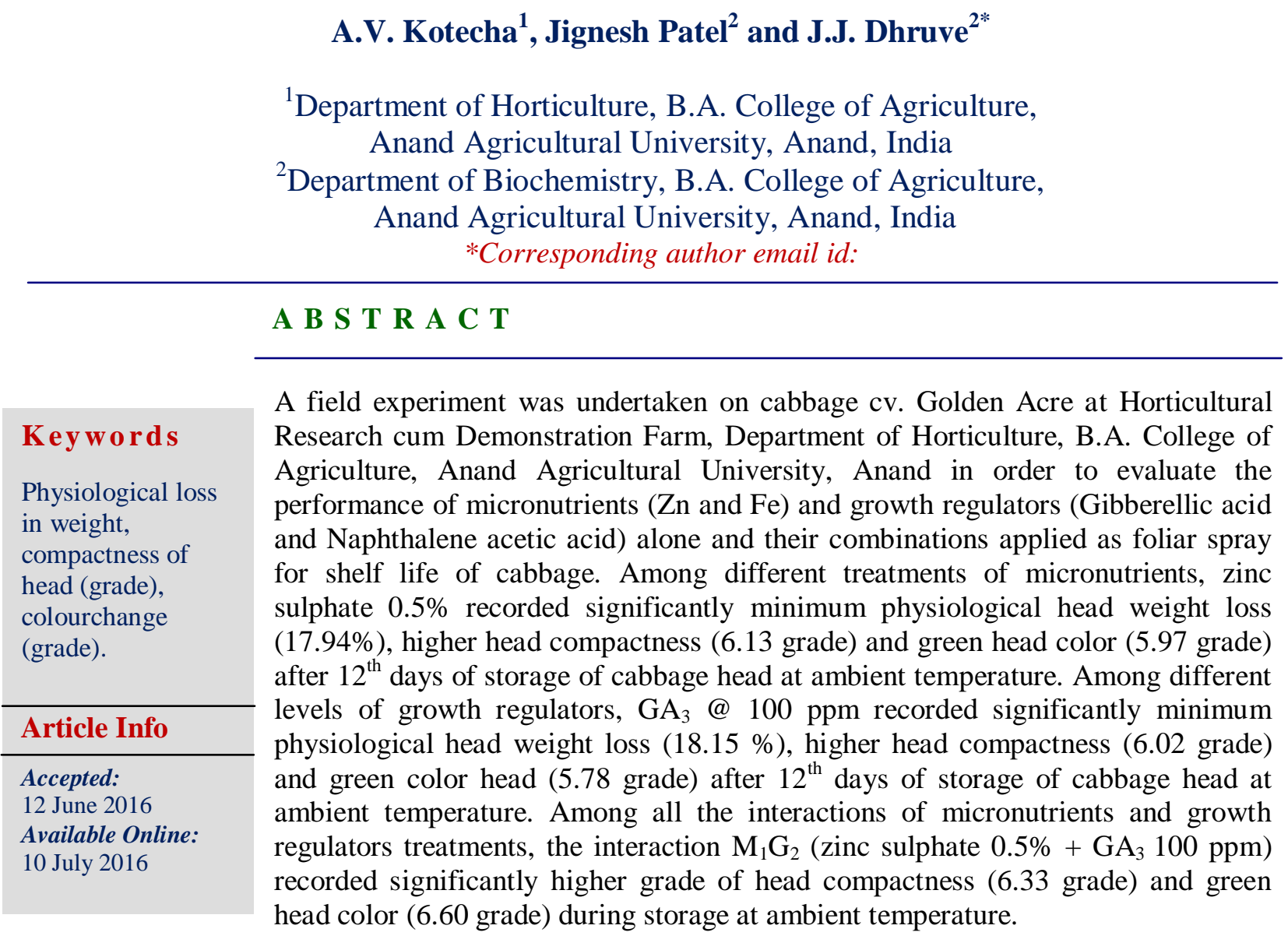

\section{Introduction}

Cabbage (Brassica oleraceaL.var. capitata) is one of the important leafy vegetable crop and used as salad, cooked, pickling as well as dehydrated vegetable. The word "Cabbage" is derived from the French word "coboche" means head. The cabbage belongs to cruciferae family. It is grown in kitchen and truck gardens. It is mostly employed as culinary and dietary article which is used alone or mixed with potatoes for vegetable purpose.
It is also used for feeding stock of chicken. The "Sauerkraut" is favourite food in Russia, Germany and U.S.A. which is made by fermenting chopped, ground or sliced cabbage in its juice with little salt added to it and from the nutritional point of view, it ranks very high. The cabbage head is rich source of vitamin A, B, C and also contains minerals. It has cooling effect and helps in preventing constipation, increase appetite, speed up digestion and very useful for patients of diabetes (Yadav et al., 2000). 
The cabbage covers about $4 \%$ of the total area under vegetables. India ranks next to China in cabbage production (Anon., 2009a). The major cabbage growing states in India are Gujarat, U.P., Orissa, W.B., Assam, Maharashtra and Karnataka. In Gujarat, cabbage crop is cultivated in almost all the districts with major cultivation in Bhavnagar, Anand, Kheda, Junagadh, Sabarkantha, Banaskantha, and Ahmedabad districts. In India, cabbage is cultivated in about an area of 309.0 thousand hectares with an annual production of 6809.0 thousand MT with the productivity of 22.04 t/ha (Anon, 2009b). In Gujarat, it is cultivated in about an area of 22.96 thousand hectares with an annual production of 404.6 thousand MT having productivity of 17.26 t/ha (Anon., 2009c). So there is tremendous scope to increase the productivity in Gujarat state.

Sillanpaa was estimated that about $30 \%$ of the agricultural soils of the world are zinc deficient (Sillanpaa, 1982). Severe zinc deficiency symptoms with corresponding decrease in yield, especially of vegetables and cereals, are found mainly in crops grown on calcareous soil of arid and semiarid regions of India (Takkar and Randhawa, 1978) as well as depression in leaf production and a leaf mottling, developed after about two weeks later. The factors which are predominantly responsible for low availability of zinc to plant roots are high $\mathrm{pH}$ and high levels of $\mathrm{CaCO}_{3}$, and low levels of organic matters and soil moisture etc.

Shelf-life of cabbage head is very important because it is used as a green salad as well as in cooked form. Shelf- life is judged on the basis of colour changes, decay and physiological weight loss. Shelf-life can be improved with the foliar spraying of growth regulators and micro nutrient. Many studies have been conducted with respect to effect of growth regulators and micro nutrients on yield and quality of cabbage but study on effect of growth regulators and micro nutrients on shelf-life of cabbage seems to be lacking. Hence, an experiment was conducted to study the effect of foliar application of growth regulators and micro nutrient on shelf-life of cabbage head.

\section{Materials and Methods}

A field experiment was conducted on cabbage cv. Golden Acre at Horticultural Research cum Demonstration Farm, Department of Horticulture, B.A. College of Agriculture, Anand Agricultural University, and Anand during the Rabi season with Factorial Randomized Block Design. The treatments comprised of three levels of micronutrients i.e. (1) Control (2) Zinc sulphate $0.5 \%$, and (3) Ferrous sulphate $0.5 \%$ and five levels of growth regulators i.e. (1) Control (2) $\mathrm{GA}_{3} @ 50 \mathrm{ppm}$, (3) $\mathrm{GA}_{3}$ @ 100 ppm, (4) NAA @ 100ppm, (5) NAA @ 200ppm) total fifteen combinations were applied as foliar sprays at $3^{\text {rd }}$ and $5^{\text {th }}$ week after transplanting of cabbage.Just after harvest, fresh weights of representative samples from each treatment was taken on physical balance and subsequent weight was measured at 9 days and 12 days for recording weight loss and it was expressed as percentage as method given by Sarma et al. (2005).

The change in compactness of head representative from each treatment was evaluated by visual scoring method (1-10) by a panel of five members. Higher score were given for compact head and lower score was given for loose head. The change of color of heads was evaluated by scoring method ( 1 to 10 ) by a panel of five members. Higher score were given for green colors head and lower score was given for yellow colored head. 


\section{Results and Discussion}

The data on physiological weight loss as influenced by micronutrient and plant growth regulator treatments during $1^{\text {st }}$ and $2^{\text {nd }}$ year as well as on pooled basis at 9days and 12days are presented in the (Table 1). The effect of micronutrient levels on physiological weight loss was found significant during $1^{\text {st }}$ and $2^{\text {nd }}$ year but nonsignificant on pooled basis. Significantly, the maximum and minimum physiological weight loss content was recorded under the treatment $\mathrm{M}_{0}(16.68 \%)$ and $\mathrm{M}_{1}(15.28 \%)$ at 9 days whereas $\mathrm{M}_{0} \quad(18.30 \%)$ and $\mathrm{M}_{1}$ (17.03\%) at 12 days respectively during 1 st year. The physiological weight loss was recorded maximum and minimum during $2^{\text {nd }}$ year under the treatment $\mathrm{M}_{1}(15.77 \%)$ and $\mathrm{M}_{0}(14.29 \%)$ at 9 days whereas $\mathrm{M}_{0}(20.32 \%)$ and $\mathrm{M}_{1}(18.85 \%)$ at 12 days. On the basis of pooled analysis the maximum PLW was recorded in $\mathrm{M}_{1}$ (15.53) at 9days whereas $\mathrm{M}_{0}$ $(19.31 \%)$ at 12 days whereas micronutrient $\mathrm{M}_{2}(15.03 \%)$ at 9 days and $\mathrm{M}_{1}(17.94 \%)$ at 12days was recorded minimum physiological weight loss.

The plant growth regulators treatments showed significant effect on physiological weight loss of cabbage head at $9^{\text {th }}$ and $12^{\text {th }}$ days after storage during $1^{\text {st }}$ and $2^{\text {nd }}$ year as well as on pooled basis, except at 12 days after storage in pooled analysis. The treatment $\mathrm{G}_{2} \quad\left(\mathrm{GA}_{3} 100 \mathrm{ppm}\right)$ recorded significantly the lowest percentage of physiological weight loss i.e. 14.49, 13.82 and 14.16 at 9 days after storage of head during $1^{\text {st }}$ and $2^{\text {nd }}$ year as well as on pooled basis and it remained at par with $\mathrm{G}_{1}\left(\mathrm{GA}_{3} 50\right.$ ppm) i.e. 15.74, 14.45 and $15.09 \%$.

At $12^{\text {th }}$ days after storage, significantly the lowest physiological weight loss i.e. $16.53 \%$ and $18.42 \%$ were recorded with the treatment $\mathrm{G}_{2}\left(\mathrm{GA}_{3} 100 \mathrm{ppm}\right)$ during $1^{\text {st }}$ and $2^{\text {nd }}$ year respectively. Tiwari et al.(2003) reported that application of $\mathrm{GA}_{3} @ 25 \mathrm{ppm}$ after transplanting significantly gave higher TSS, while physiological weight loss was lowest with the application of NAA @ 50 ppm as compared to control in onion.

The compactness of head at $9^{\text {th }}$ and $12^{\text {th }}$ days after storage during $1^{\text {st }}$ and $2^{\text {nd }}$ year as well as in pooled basis were presented in table2.The treatment of micronutrients $\mathrm{M}_{1}$ (zinc sulphate $0.5 \%$ ) recorded significantly the highest grade i.e. 7.18, 6.98, 7.08 and 6.18, $6.09,6.13$ at $9^{\text {th }}$ and $12^{\text {th }}$ days after storage of head during $1^{\text {st }}$ and $2^{\text {nd }}$ year as well as on pooled basis respectively Whereas the treatment of plant growth regulator $\mathrm{G}_{2}\left(\mathrm{GA}_{3}\right.$ $100 \mathrm{ppm}$ ) recorded highest grade of compactness of head i.e. 7.07, 6.73, 6.90 and $6.07,5.99,6.02$, at 9 and 12 days after storage during $1^{\text {st }}$ and $2^{\text {nd }}$ year as well as on pooled basis respectively.

After $9^{\text {th }}$ and $12^{\text {th }}$ days, the interaction effect between micronutrient and plant growth regulator for compactness of head were found significantly during $1^{\text {st }}$ and $2^{\text {nd }}$ year. At $9^{\text {th }}$ days after storage (Table-3 \& 4), interaction of $\mathrm{M}_{1} \mathrm{G}_{2}$ (zinc sulphate $0.5 \%+$ $\mathrm{GA}_{3} 100 \mathrm{ppm}$ ) recorded significantly higher grade during $1^{\text {st }}$ year (7.40) and during $2^{\text {nd }}$ year(7.42). At the same on $12^{\text {th }}$ days after storage, interaction of $\mathrm{M}_{1} \mathrm{G}_{2}$ (zinc sulphate $0.5 \%+\mathrm{GA}_{3} 100 \mathrm{ppm}$ )recorded higher grade of compactness of head during $1^{\text {st }}$ year (6.40) and during $2^{\text {nd }}$ year (6.27) and 6.68 during on pooled basis respectively. Chhonkar and Singh (1964) conducted an experiment on 'Late Drumhead' variety of cabbage at Sabour (Bihar). 
Table.1 Effects of micronutrients and growth regulators on physiological weight loss (\%) of cabbage head at 9 th and 12th days of storage

\begin{tabular}{|c|c|c|c|c|c|c|}
\hline \multirow[t]{2}{*}{ Treatment } & \multicolumn{3}{|c|}{$\begin{array}{l}\text { Physiological weight loss of } \\
\text { cabbage head at ( } 9 \text { DAS ) }\end{array}$} & \multicolumn{3}{|c|}{$\begin{array}{c}\text { Physiological weight loss of cabbage } \\
\text { head at ( } 12 \text { DAS ) }\end{array}$} \\
\hline & $1^{\text {st }}$ Year & $2^{\text {nd }}$ Year & Pooled & $1^{\text {st }}$ Year & $2^{\text {nd }}$ year & Pooled \\
\hline \multicolumn{7}{|c|}{ Micronutrients } \\
\hline $\mathrm{M}_{0}$ & 16.68 & 14.29 & 15.49 & 18.30 & 20.32 & 19.31 \\
\hline $\mathrm{M}_{1}$ & 15.28 & 15.77 & 15.53 & 17.03 & 18.85 & 17.94 \\
\hline $\mathrm{M}_{2}$ & 15.70 & 14.47 & 15.03 & 17.92 & 20.06 & 18.99 \\
\hline S.Em \pm & 0.38 & 0.40 & 0.72 & 0.28 & 0.42 & 0.25 \\
\hline C.D. $5 \%$ & 1.11 & 1.16 & NS & 0.82 & 1.21 & 0.72 \\
\hline \multicolumn{7}{|c|}{ Growth regulators } \\
\hline $\mathrm{G}_{0}$ & 17.07 & 15.45 & 16.26 & 18.63 & 20.42 & 19.52 \\
\hline $\mathrm{G}_{1}$ & 15.74 & 14.45 & 15.09 & 17.88 & 19.83 & 18.18 \\
\hline $\mathrm{G}_{2}$ & 14.49 & 13.82 & 14.16 & 16.53 & 18.42 & 18.15 \\
\hline $\mathrm{G}_{3}$ & 16.14 & 14.30 & 15.22 & 18.08 & 19.13 & 18.60 \\
\hline $\mathrm{G}_{4}$ & 15.82 & 16.20 & 16.01 & 17.62 & 20.92 & 19.27 \\
\hline S.Em \pm & 0.50 & 0.52 & 0.36 & 0.37 & 0.54 & 0.64 \\
\hline C.D. $5 \%$ & 1.43 & 1.50 & 1.01 & 1.06 & 1.56 & NS \\
\hline Sig. Int. & & MXG & - & - & MXG & - \\
\hline C.V. \% & 9.37 & 10.45 & 9.89 & 6.20 & 8.19 & 7.38 \\
\hline
\end{tabular}

Table.2 Effects of micronutrients and growth regulators on compactness of head (grade) at 9th and 12 th days of storage

\begin{tabular}{|c|c|c|c|c|c|c|}
\hline \multirow{2}{*}{ Treatment } & \multicolumn{3}{|c|}{ Compactness of head at ( 9 DAS ) } & \multicolumn{3}{|c|}{ Compactness of head at (12 DAS ) } \\
\hline & $1^{\text {st }}$ Year & $2^{\text {nd }}$ Year & Pooled & $1^{\text {st }}$ Year & $2^{\text {nd }}$ Year & Pooled \\
\hline \multicolumn{7}{|c|}{ Micronutrients } \\
\hline $\mathrm{M}_{0}$ & 6.40 & 6.00 & 6.20 & 5.39 & 5.38 & 5.39 \\
\hline $\mathrm{M}_{1}$ & 7.18 & 6.98 & 7.08 & 6.18 & 6.09 & 6.13 \\
\hline $\mathrm{M}_{2}$ & 6.84 & 6.71 & 6.78 & 5.84 & 5.73 & 5.79 \\
\hline S.Em \pm & 0.03 & 0.04 & 0.07 & 0.03 & 0.05 & 0.03 \\
\hline C.D.5\% & 0.09 & 0.14 & 0.21 & 0.07 & 0.15 & 0.08 \\
\hline \multicolumn{7}{|c|}{ Growth regulators } \\
\hline $\mathrm{G}_{0}$ & 6.60 & 6.60 & 6.60 & 5.60 & 5.54 & 5.57 \\
\hline $\mathrm{G}_{1}$ & 6.73 & 6.40 & 6.57 & 5.73 & 5.73 & 5.73 \\
\hline $\mathrm{G}_{2}$ & 7.07 & 6.73 & 6.90 & 6.07 & 5.99 & 6.02 \\
\hline $\mathrm{G}_{3}$ & 6.80 & 6.47 & 6.63 & 5.79 & 5.70 & 5.74 \\
\hline $\mathrm{G}_{4}$ & 6.83 & 6.61 & 6.72 & 5.83 & 5.71 & 5.77 \\
\hline S.Em \pm & 0.03 & 0.06 & 0.07 & 0.03 & 0.06 & 0.04 \\
\hline C.D. $5 \%$ & 0.10 & 0.18 & NS & 0.09 & 0.18 & 0.16 \\
\hline Sig. Int. & $\mathrm{MXG}$ & $\mathrm{MXG}$ & - & $\mathrm{MXG}$ & $\mathrm{MXG}$ & MXG \\
\hline C.V. $\%$ & 2.49 & 3.85 & 3.25 & 2.70 & 3.31 & 2.63 \\
\hline
\end{tabular}


Table.3 Interaction effect of micronutrients and growth regulators on compactness of head (grade) at 9th days of storage

\begin{tabular}{|c|c|c|c|c|c|}
\hline \multirow{2}{*}{ Micronutrients } & \multicolumn{5}{|c|}{ Growth regulators } \\
\hline & $\mathbf{G}_{\mathbf{0}}$ & $\mathbf{G}_{1}$ & $\mathbf{G}_{2}$ & $\mathbf{G}_{3}$ & $\mathbf{G}_{4}$ \\
\hline \multicolumn{6}{|c|}{ First Year } \\
\hline $\mathrm{M}_{0}$ & 6.00 & 6.30 & 6.80 & 6.50 & 6.40 \\
\hline $\mathrm{M}_{1}$ & 7.00 & 7.10 & 7.40 & 7.10 & 7.30 \\
\hline $\mathrm{M}_{2}$ & 6.80 & 6.80 & 7.00 & 6.80 & 6.80 \\
\hline $\mathrm{S} . \mathrm{Em} \pm$ & \multicolumn{5}{|c|}{0.06} \\
\hline C.D.5\% & \multicolumn{5}{|c|}{0.17} \\
\hline \multicolumn{6}{|c|}{ Second Year } \\
\hline $\mathrm{M}_{0}$ & 5.30 & 6.00 & 5.80 & 6.50 & 6.40 \\
\hline $\mathrm{M}_{1}$ & 7.00 & 7.10 & 7.42 & 6.10 & 7.30 \\
\hline $\mathrm{M}_{2}$ & 6.80 & 6.80 & 7.00 & 6.80 & 6.13 \\
\hline S.Em \pm & \multicolumn{5}{|c|}{0.11} \\
\hline C.D.5\% & \multicolumn{5}{|c|}{0.31} \\
\hline
\end{tabular}

Table.4 Interaction effect of micronutrients and growth regulators on compactness of head (grade) at 12th days of storage

\begin{tabular}{|c|c|c|c|c|c|}
\hline \multirow{2}{*}{ Micronutrients } & \multicolumn{5}{|c|}{ Growth regulators } \\
\hline & $\mathbf{G}_{\mathbf{0}}$ & $\mathbf{G}_{1}$ & $\mathbf{G}_{2}$ & $\mathbf{G}_{3}$ & $\mathbf{G}_{4}$ \\
\hline \multicolumn{6}{|c|}{ First Year } \\
\hline $\mathrm{M}_{0}$ & 5.00 & 5.30 & 5.80 & 5.47 & 5.40 \\
\hline $\mathrm{M}_{1}$ & 6.00 & 6.10 & 6.40 & 6.10 & 6.30 \\
\hline $\mathrm{M}_{2}$ & 5.80 & 5.80 & 6.00 & 5.80 & 5.80 \\
\hline S.Em \pm & \multicolumn{5}{|c|}{0.06} \\
\hline C.D. $5 \%$ & \multicolumn{5}{|c|}{0.17} \\
\hline \multicolumn{6}{|c|}{ Second Year } \\
\hline $\mathrm{M}_{0}$ & 4.93 & 5.30 & 5.80 & 5.47 & 5.40 \\
\hline $\mathrm{M}_{1}$ & 5.90 & 6.10 & 6.27 & 6.03 & 6.17 \\
\hline $\mathrm{M}_{2}$ & 5.80 & 5.80 & 5.90 & 5.60 & 5.57 \\
\hline S.Em+ & \multicolumn{5}{|c|}{0.11} \\
\hline C.D. $5 \%$ & \multicolumn{5}{|c|}{0.32} \\
\hline \multicolumn{6}{|c|}{ Pooled } \\
\hline $\mathrm{M}_{0}$ & 4.97 & 5.30 & 5.80 & 5.47 & 5.40 \\
\hline $\mathrm{M}_{1}$ & 5.95 & 6.10 & 6.68 & 6.07 & 6.23 \\
\hline $\mathrm{M}_{2}$ & 5.80 & 5.80 & 5.95 & 5.70 & 6.33 \\
\hline S.Em \pm & \multicolumn{5}{|c|}{0.06} \\
\hline C.D.5\% & \multicolumn{5}{|c|}{0.18} \\
\hline
\end{tabular}


Table.5 Effects of micronutrients and growth regulators on colour change (grade) of cabbage head at 9 th and 12th days of storage

\begin{tabular}{|c|c|c|c|c|c|c|}
\hline \multirow{2}{*}{ Treatment } & \multicolumn{3}{c|}{$\begin{array}{c}\text { Color change of head at } \\
(\mathbf{9} \text { DAS })\end{array}$} & \multicolumn{3}{c|}{$\begin{array}{c}\text { Oolor change of head at } \\
(\mathbf{1 2} \text { DAS })\end{array}$} \\
\cline { 2 - 7 } & $\mathbf{1}^{\text {st }}$ Year & $\mathbf{2}^{\text {nd }}$ Year & Pooled & $\mathbf{1}^{\text {st }}$ Year & $\mathbf{2}^{\text {nd }}$ Year & Pooled \\
\hline \multicolumn{7}{|c|}{ Micronutrients } \\
\hline $\mathrm{M}_{0}$ & 6.16 & 5.69 & 5.92 & 5.01 & 4.88 & 4.94 \\
\hline $\mathrm{M}_{1}$ & 6.61 & 6.37 & 6.49 & 5.79 & 6.15 & 5.97 \\
\hline $\mathrm{M}_{2}$ & 6.37 & 6.34 & 6.35 & 5.65 & 6.12 & 5.88 \\
\hline S.Em+ & 0.10 & 0.09 & 0.18 & 0.09 & 0.09 & 0.18 \\
\hline C.D.5\% & 0.28 & 0.26 & 0.54 & 0.25 & 0.25 & 0.53 \\
\hline \multicolumn{7}{|c|}{ Growth regulators } \\
\hline $\mathrm{G}_{0}$ & 6.03 & 5.79 & 5.91 & 5.12 & 5.63 & 5.38 \\
\hline $\mathrm{G}_{1}$ & 6.37 & 6.14 & 6.26 & 5.33 & 5.43 & 5.38 \\
\hline $\mathrm{G}_{2}$ & 6.53 & 6.37 & 6.45 & 5.72 & 5.86 & 5.78 \\
\hline $\mathrm{G}_{3}$ & 6.46 & 6.32 & 6.39 & 5.50 & 5.82 & 5.77 \\
\hline $\mathrm{G}_{4}$ & 6.51 & 6.03 & 6.27 & 5.73 & 5.83 & 5.78 \\
\hline S.Em \pm & 0.12 & 0.11 & 0.08 & 0.11 & 0.09 & 0.07 \\
\hline C.D.5\% & 0.36 & 0.33 & 0.24 & 0.32 & 0.25 & 0.20 \\
\hline Sig. Int. & MXG & MXG & - & MXG & MXG & - \\
\hline C.V. \% & 5.79 & 5.58 & 5.69 & 6.14 & 4.50 & 5.35 \\
\hline
\end{tabular}

Table.6 Interaction effect of micronutrients and growth regulators on color change of head (grade) at 9th days of storage

\begin{tabular}{|c|c|c|c|c|c|}
\hline \multirow{2}{*}{ Micronutrients } & \multicolumn{5}{|c|}{ Growth regulators } \\
\hline & $\mathbf{G}_{0}$ & $\mathbf{G}_{1}$ & $\mathbf{G}_{2}$ & $\mathbf{G}_{\mathbf{3}}$ & $\mathbf{G}_{4}$ \\
\hline \multicolumn{6}{|c|}{ First Year } \\
\hline $\mathrm{M}_{0}$ & 5.67 & 6.47 & 6.40 & 6.17 & 6.10 \\
\hline $\mathrm{M}_{1}$ & 6.17 & 6.27 & 7.37 & 6.67 & 6.60 \\
\hline $\mathrm{M}_{2}$ & 6.27 & 6.37 & 5.83 & 6.53 & 6.83 \\
\hline S.Em \pm & \multicolumn{5}{|c|}{0.21} \\
\hline C.D.5\% & \multicolumn{5}{|c|}{0.62} \\
\hline \multicolumn{6}{|c|}{ Second Year } \\
\hline $\mathrm{M}_{0}$ & 5.33 & 5.83 & 5.80 & 5.57 & 5.90 \\
\hline $\mathrm{M}_{1}$ & 5.63 & 6.30 & 6.86 & 6.83 & 6.36 \\
\hline $\mathrm{M}_{2}$ & 6.40 & 6.30 & 6.60 & 6.57 & 5.83 \\
\hline S.Em \pm & \multicolumn{5}{|c|}{0.20} \\
\hline C.D. $5 \%$ & \multicolumn{5}{|c|}{0.57} \\
\hline
\end{tabular}


Table.7 Interaction effect of micronutrient and growth regulators on color change of head (grade) at 12th days of storage

\begin{tabular}{|c|c|c|c|c|c|}
\hline \multirow{2}{*}{ Micronutrients } & \multicolumn{5}{|c|}{ Growth regulators } \\
\hline & $\mathbf{G}_{\mathbf{0}}$ & $\mathbf{G}_{1}$ & $\mathbf{G}_{2}$ & $\mathbf{G}_{3}$ & $\mathbf{G}_{4}$ \\
\hline \multicolumn{6}{|c|}{ First Year } \\
\hline $\mathbf{M}_{0}$ & 4.70 & 5.23 & 5.00 & 4.97 & 5.13 \\
\hline $\mathrm{M}_{1}$ & 5.17 & 5.27 & 6.60 & 6.57 & 6.27 \\
\hline $\mathrm{M}_{2}$ & 5.50 & 5.50 & 5.83 & 5.60 & 5.80 \\
\hline S.Em \pm & \multicolumn{5}{|c|}{0.19} \\
\hline C.D. $5 \%$ & \multicolumn{5}{|c|}{0.56} \\
\hline \multicolumn{6}{|c|}{ Second Year } \\
\hline $\mathrm{M}_{0}$ & 4.26 & 4.70 & 4.93 & 5.10 & 5.40 \\
\hline $\mathrm{M}_{1}$ & 6.00 & 6.10 & 6.53 & 6.13 & 5.97 \\
\hline $\mathrm{M}_{2}$ & 6.20 & 5.93 & 6.10 & 6.23 & 6.13 \\
\hline S.Em+ & \multicolumn{5}{|c|}{0.15} \\
\hline C.D. $5 \%$ & \multicolumn{5}{|c|}{0.43} \\
\hline
\end{tabular}

The plants were sprayed with $\mathrm{GA}_{3} @ 5$ and $10 \mathrm{ppm}$ at $2^{\text {nd }}$ and $3^{\text {rd }}$ week after transplanting. The results showed that both the $\mathrm{GA}_{3}$ concentrations increased number of inner leaves, head diameter, compactness of head, percentage of head formation, earliest of head formation and yield (215.2 q/acre) as compared to control.

Results of the colour change of head at $9^{\text {th }}$ and $12^{\text {th }}$ days after storage during $1^{\text {st }}$ and $2^{\text {nd }}$ year as well as in pooled basis were presented in table-5, $6 \& 7$. Micronutrients effects on colour of head at $9^{\text {th }}$ and $12^{\text {th }}$ days after storage were found significant during $1^{\text {st }}$ year, $2^{\text {nd }}$ year and in pooled basis (Table 4.14). The treatment $M_{1}$ (zinc sulphate $0.5 \%$ ) recorded highest grade (i.e. 6.61, $6.37,6.49$ at 9days after storage and 5.79, $6.15,5.97$ at 12 days after storage during $1^{\text {st }}$ and $2^{\text {nd }}$ year as well as on pooled basis respectively. Among plant growth regulators, treatment $\mathrm{G}_{2} \quad\left(\mathrm{GA}_{3} 100 \mathrm{ppm}\right)$ recorded higher grade of colour of head i.e. $6.53,6.37,6.45$ at $9^{\text {th }}$ days and 5.72, 5.86, 5.78 at $12^{\text {th }}$ days after storage of head during $1^{\text {st }}$ and $2^{\text {nd }}$ year as well as in pooled basis. Interactions effect between micronutrient and plant growth regulator $\mathrm{M}_{1} \mathrm{G}_{2}$ (zinc sulphate $0.5 \%$ + GA3 $100 \mathrm{ppm}$ ) on colour change of head were found significant at $9^{\text {th }}$ and $12^{\text {th }}$ days after storage during both the individual $1^{\text {st }}$ and $2^{\text {nd }}$ year. Sharma et al. (2005) studied that the spray of $0.5 \%$ zinc sulphate recorded the highest root length and minimum mean weight loss of cabbage head and superior for maintenance of head colour during storage.

\section{References}

Annonymous. 2009a. Cabbage- www. Free encyclopedia.

Annonymous. 2009b. Area, production and yield of horticulture and plantation crop. www. Agricultural statistics at a glance.

Annonymous. 2009c. Area and production of various horticultural crops. www. agri.gujarat.gov.in

Chhonkar, V.S., Singh, R. 1964. Effect of gibberellic acid on growth, yield and quality of cabbage (Brassica oleraceavar. capitata L.). Indian J. Hort., 21: 57-63. 
Sarma, P., Goswami, R.K., Deka, B.C. 2005. Effect of foliar application of micronutrients on shelf life of cabbage. Indian J. Hort., 62(2): 16062.

Sillanpaa, M. 1982. FAO Soils Bulletin 48, Food and Agriculture Organization of United Nations, Rome.

Takkar, P.N., Randhawa, N.S. 1978. Fert. News, 18: 3.
Tiwari, R.S., Agarwal, A., Sengar, S.C. 1993. Effect of bio-regulators on growth, bulb yield, quality and storability of onion cv. Pusa red. Indian J. Plant Physiol., 8(4): 411-13.

Yadav, R.L. Dhaka, R.S., Fageria, M.S. 2000. Effect of $\mathrm{GA}_{3}$, NAA and succcinic acid on growth and yield of cabbage cv. Golden Acre. Haryana J. Hort. Sci., 20(20): 269-70.

\section{How to cite this article:}

Kotecha, A.V., Jignesh Patel and J.J. Dhruve. 2016. Influence of Micronutrients and Growth Regulators on Shelf-life of Cabbage. Int.J.Curr.Microbiol.App.Sci. 5(7): 329-336. doi: http://dx.doi.org/10.20546/ijcmas.2016.507.035 